\title{
Feature-Weighted Mountain Method with Its Application to Color Image Segmentation
}

\author{
Wen-Liang Hung ${ }^{1}$, Miin-Shen Yang ${ }^{2}$, Jian Yu ${ }^{3}$, Chao-Ming Hwang ${ }^{4}$ \\ ${ }^{1}$ Department of Applied Mathematics, National Hsinchu University of Education, Hsin-Chu, Taiwan \\ wlhung@mail.nhcue.edu.tw \\ ${ }^{2}$ Department of Applied Mathematics, Chung Yuan Christian University, Chung-Li, Taiwan \\ ${ }^{3}$ Department of Computer Science, Beijing Jiaotong University, Beijing, China \\ ${ }^{4}$ Department of Applied Mathematics, Chinese Culture University Yangminshan, Taipei, Taiwan
}

\begin{abstract}
In this paper, we propose a feature-weighted mountain clustering method. The proposed method can work well when there are noisy feature variables and could be useful for obtaining initial estimat of cluster centers for other clustering algorithms. Results from color image segmentation illustrate the proposed method actually produces better segmentation than previous methods.
\end{abstract}

Keywords: Mountain method; Feature weight; Color image segmentation.

\section{Introduction}

Cluster analysis is a method of clustering a data set into groups. It is an approach to unsupervised learning and one of major techniques used in pattern recognition. Yager and Filev [7] proposed a simple and effective algorithm, called the mountain method, as an approximate clustering technique. Chiu [1] modified the original mountain method by considering the mountain function on the data points instead of the grid nodes. The approach is based on the density estimation in feature space with the highest potential value chosen as a cluster center and then new density estimation is created for the extraction of the next cluster center. The process is repeated until a stopping condition is satisfied. This method can be used to obtain initial guesses of cluster centers for other clustering algorithms.

Yang and $\mathrm{Wu}[6]$ created another modified mountain clustering algorithm. The proposed algorithm can automatically estimate the parameters in the modified mountain function in accordance with the structure of the data set based on the correlation of self-comparison method. However, the modified mountain function treats all the features of equal importance. In practice, there may be some noisy variables in the data set in which these variables may influence the performance of clustering results. To solve this problem, we propose a modified algorithm, called feature-weighted mountain method. This method can work well for noisy feature variables.

The remainder of this paper is organized as follows. In Section 2, we first describe Yang and Wu's [6] mountain clustering algorithm. We then present the feature-weighted mountain method. For estimating feature weights, we propose an attribute weight method based on a variation approach. Image segmentation is an important step for many image processing and computer vision. The proposed algorithm is used to obtain approximate cluster centers and applied it to color im- age segmentation. The segmentation results with comparisons are given in Section 3. Finally, we make our conclusions in Section 4.

\section{The proposed feature-weighted mountain method}

Let $\boldsymbol{X}=\left\{X_{1}, \cdots, X_{n}\right\}$ be a data set where $X_{i}=$ $\left(x_{i 1}, x_{i 2}, \ldots, x_{i p}\right), i=1, \cdots, n$ are feature vectors in $p$ dimensional Euclidean space $R^{p}$. Yang and $\mathrm{Wu}[6]$ modified the mountain method (cf. [7]), and proposed the modified mountain function for each data vector $X_{i}$ on all data points as

$$
\begin{aligned}
M_{1}\left(X_{i}\right)= & \sum_{\substack{j=1 \\
i=1, \cdots, n}}^{n} \exp \left(-m\left\|X_{i}-X_{j}\right\|^{2} / \sigma^{2}\right), \\
& i=1,
\end{aligned}
$$

where

$$
\left\|X_{i}-X_{j}\right\|^{2}=\sum_{l=1}^{p}\left(x_{i l}-x_{j l}\right)^{2}
$$

is the Euclidean distance between the $i$ th data point $X_{i}$ and the $j$ th data point $X_{j}, \sigma^{2}=\sum_{i=1}^{n}\left\|X_{i}-\bar{X}\right\|^{2} / n$, with $\bar{X}=\sum_{i=1}^{n} X_{i} / n$. The parameter $m$ in Eq. (1) is to determine the approximate density shape of the data set. Thus, the role of $m$ is similar to the bandwith in a kernel density estimate defined on the data set $\boldsymbol{X}$. The kernel density estimate with kernel $K$ and bandwith $h$ is defined by

$$
\hat{f}(x)=\frac{1}{n \sigma^{p} h^{p}} \sum_{j=1}^{n} K\left(\frac{x-X_{j}}{\sigma h}\right) .
$$

In this section, we consider the standard multivariate normal density function

$$
K(x)=(2 \pi)^{-p / 2} \exp \left(\frac{-1}{2}\|x\|^{2}\right) .
$$


Thus,

$$
\hat{f}(x)=\frac{1}{n \sigma^{p} h^{p}(2 \pi)^{p / 2}} \sum_{j=1}^{n} \exp \left(\frac{-1}{2 h \sigma^{2}}\left\|x-X_{j}\right\|^{2}\right) .
$$

If the underlying density is the multivariate normal, then the optimal bandwith is given by (cf. [5])

$$
h_{o p t}=A \cdot n^{-1 /(p+4)},
$$

where

$$
A= \begin{cases}0.96, & \text { if } p=2 \\ \left(\frac{4}{2 p+1}\right)^{1 /(p+4)}, & \text { if } p>2 .\end{cases}
$$

Compared the estimated density function Eq. (2) with the mountain function Eq. (1), we obtain

$$
m=\frac{n^{2 /(p+4)}}{2 A^{2}} \text {. }
$$

We then choose

$$
m_{0}=\left[\frac{n^{2 /(p+4)}}{2 A^{2}}\right]
$$

as the initial value in Yang and Wu's correlation selfcomparison algorithm. To implement this algorithm, the modified mountain method is rewritten as

$$
M_{1}^{m_{0}}\left(X_{i}\right)=\sum_{j=1}^{n} \exp \left(-m_{0}\left\|X_{i}-X_{j}\right\|^{2} / \sigma^{2}\right),
$$

and

$$
M_{1}^{m_{t}}\left(X_{i}\right)=\sum_{j=1}^{n} \exp \left(-m_{t}\left\|X_{i}-X_{j}\right\|^{2} / \sigma^{2}\right),
$$

where $m_{t}=m_{0}+t, t=1,2,3, \cdots$. The correlation selfcomparison procedure is summarized as follows.

S1. Set $t=1$ and $\rho=0.99$.

S2. Calculate the correlation between $\left\{M_{1}^{m_{(t-1)}}\left(X_{i}\right) \mid i=\right.$ $1, \cdots, n\}$ and $\left\{M_{1}^{m_{t}}\left(X_{i}\right) \mid i=1, \cdots, n\right\}$.

S3. IF the correlation is greater than or equal the specified $\rho$,

THEN choose $\left\{M_{1}^{m_{(t-1)}}\left(X_{i}\right)\right.$ to be the modified mountain function;

ELSE $t=t+1$ and GOTO S2

After the parameter $m$ is estimated by the correlation self-comparison algorithm, the modified mountain function is obtained. Next, we will search for the $k$ th cluster center using the following modified revised mountain function

$$
\begin{aligned}
M_{k}\left(X_{i}\right)= & M_{k-1}\left(X_{i}\right)- \\
& M_{k-1}\left(X_{i}\right) \cdot \exp \left(-\left\|X_{i}-X_{k-1}^{*}\right\|^{2} / \sigma^{2}\right), \\
& k=2,3, \cdots
\end{aligned}
$$

where $X_{k-1}^{*}$ is the $(k-1)$ th cluster center which satisfies

$$
M_{k-1}\left(X_{k-1}^{*}\right)=\max _{i}\left\{M_{k-1}\left(X_{i}\right)\right\}, k=2,3, \cdots .
$$

To determine the stopping condition for the modified mountain method, Yang and $\mathrm{Wu}[6]$ proposed a validity function as follows:

$$
M V(c)=\sum_{k=2}^{c} \operatorname{pot}(k), c=2,3, \cdots, n-1
$$

where $c$ is the number of clusters. The function $\operatorname{pot}(k)$ is the potential of the $k$ th cluster center $X_{k}^{*}$ and is defined as

$$
\begin{aligned}
\operatorname{pot}(k)= & M_{1}\left(X_{k}^{*}\right) \cdot \frac{M_{1}\left(X_{k}^{*}\right)}{M_{1}\left(X_{1}^{*}\right)}- \\
& n \cdot \exp \left(-m d_{k}^{2} / \sigma^{2}\right), k=2,3, \cdots
\end{aligned}
$$

where $d_{k}^{2}$ is the minimum distance among $X_{k}^{*}$ and all $(k-1)$ previous identified cluster centers, i.e.,

$d_{k}^{2}=\min \left\{\left\|X_{k}^{*}-X_{k-1}^{*}\right\|^{2},\left\|X_{k}^{*}-X_{k-2}^{*}\right\|^{2}, \cdots,\left\|X_{k}^{*}-X_{1}^{*}\right\|^{2}\right\}$.

Thus, Yang and Wu's [6] modified mountain clustering algorithm is summarized as follows:

S1. Obtain the modified mountain function using the correlation self-comparison algorithm.

$\mathrm{S} 2$. Fix the $k$ th cluster center $X_{k}^{*}$ using the modified revised mountain function Eq. (3) and condition Eq. (4).

S3. Calculate $M V(c), c=2,3, \cdots, n-1$.

S4. Choose the cluster number estimate with the maximum value of $M V(c)$ and select these $c$ extracted cluster centers.

From Eq. (1), the modified mountain function treats all features equal important. In practice, there may exist some noise variables in the data set and these variables may influence the performance of clustering results. Figs.1.1 and 2.1 present an artificial data set shown in Table 1 (cf. Table 1 in Huang et al. [2]) to demonstrate that the performance of Yang and Wu's [6] modified mountain clustering algorithm is affected by diverse (or noise) variables. Fig. 1.1 shows the subspace of $(x 0, x 1)$ with three normally distributed clusters. Fig. 2.1 presents the subspace of $(x 0, x 4)$ with uniformly distributed noise points. Figs. 1.1 and 1.2 demonstrate Yang and Wu's [6] modified mountain function with a good and poor density shape when the data set with normally distributed and the noise variable exists, respectively. These results illustrate that the noise variable influences the performance of clustering results. 
Table 1. Centroid and Standard Deviations of Clusters in Different Variables

\begin{tabular}{|c|c|c|c|}
\hline Cluster & Cluster centroid & Standard deviations & No. of points \\
\hline 1 & $(0.547,0.728,0.424,0.492,0.561)$ & $(0.054,0.044,0.071,0.288,0.302)$ & 100 \\
2 & $(0.299,0.585,0.318,0.555,0.455)$ & $(0.061,0.044,0.069,0.269,0.274)$ & 100 \\
3 & $(0.422,0.452,0.636,0.520,0.536)$ & $(0.055,0.050,0.075,0.263,0.274)$ & 100 \\
\hline
\end{tabular}

To overcome this problem, we proposed a featureweight mountain function as follows. Let $W=$ $\left(w_{1}, \cdots, w_{p}\right)$ be the weights for $p$ variables. According to Huang et al's [2] W-k-means algorithm, the weighted mountain function may be defined as

$$
\begin{aligned}
M_{1}^{w}\left(X_{i}\right)= & \sum_{j=1}^{n} \exp \left(-\gamma\left\|X_{i}-X_{j}\right\|_{w}^{2} / \sigma^{2}\right), \\
& i=1, \cdots, n
\end{aligned}
$$

where $\gamma>0$,

$$
\sum_{l=1}^{p} w_{l}=1,0 \leq w_{l} \leq 1
$$

and

$$
\left\|X_{i}-X_{j}\right\|_{w}^{2}=\sum_{l=1}^{p} w_{l}^{\beta}\left(x_{i l}-x_{j l}\right)^{2},
$$

where $\beta<0$ or $\beta>1$. The weighted revised mountain function

$$
\begin{aligned}
M_{k}^{w}\left(X_{i}\right)= & M_{k-1}^{w}\left(X_{i}\right)- \\
& M_{k-1}^{w}\left(X_{i}\right) \cdot \exp \left(\left\|X_{i}-X_{k-1}^{*}\right\|_{w}^{2} / \sigma^{2}\right), \\
& k=2, \cdots
\end{aligned}
$$

where $X_{k-1}^{*}$ is the $(k-1)$ th cluster center which satisfies

$$
M_{k-1}^{w}\left(X_{k-1}^{*}\right)=\max _{i}\left\{M_{k-1}^{w}\left(X_{i}\right)\right\}, k=2,3, \cdots .
$$

To determine the stopping condition for the weighted mountain method, we proposed a validity function as follows:

$$
V(c)=\sum_{k=2}^{c} \operatorname{pot}^{w}(k), c=2, \cdots, n-1 .
$$

The function $\operatorname{pot}^{w}(k)$ is the weighted potential of the $k$ th cluster center $X_{k}^{*}$ and is defined as

$$
\begin{aligned}
\operatorname{pot}^{w}(k)= & M_{1}^{w}\left(X_{k}^{*}\right) \cdot \frac{M_{1}^{w}\left(X_{k}^{*}\right)}{M_{1}^{w}\left(X_{1}^{*}\right)}- \\
& n \cdot \exp \left(-\gamma\left(d_{k}^{w}\right)^{2} / \sigma^{2}\right), k=2, \cdots
\end{aligned}
$$

where $\left(d_{k}^{w}\right)^{2}$ is the minimum weighted distance among $X_{k}^{*}$ and all $(k-1)$ previous identified cluster centers, i.e.,

$$
\begin{aligned}
\left(d_{k}^{w}\right)^{2}= & \min \left\{\left\|X_{k}^{*}-X_{k-1}^{*}\right\|_{w}^{2},\left\|X_{k}^{*}-X_{k-2}^{*}\right\|_{w}^{2}, \cdots,\right. \\
& \left.\left\|X_{k}^{*}-X_{1}^{*}\right\|_{w}^{2}\right\} .
\end{aligned}
$$

\subsection{Variation approach to attribute weight}

It is known that variation plays an important role in statistics. Let us start from scratch and devise a measure of variability that uses a random sample of size $n$, $\left\{x_{1}, \cdots, x_{n}\right\} \subset R$, where $R$ is the one dimensional Euclidean space, it would logically indicate what we construct should measure how the data vary from average. The sample standard deviation, $s$, is an usual measure of variability, defined as

$$
s=\sqrt{\frac{\sum_{i=1}^{n}\left(x_{i}-\bar{x}\right)^{2}}{n-1}}, \bar{x}=\frac{1}{n} \sum_{i=1}^{n} x_{i} .
$$

In practice, Karl Pearson's coefficient of variation (CV) has been used extensively, defined by

$$
c v=\frac{s}{\bar{x}} \text {. }
$$

On the other hand, if we have a random sample $\boldsymbol{X}=$ $\left\{X_{1}, \cdots, X_{n}\right\} \subset R^{p}$ and $X_{i}=\left(x_{i 1}, \cdots, x_{i p}\right)$ represents the $i$ th sample, then the $\mathrm{CV}$ of the $l$ th attribute is defined as

$$
c v_{l}=\frac{\sqrt{\sum_{i=1}^{n}\left(x_{i l}-\bar{x}_{l}\right)^{2} /(n-1)}}{\bar{x}_{l}}, \bar{x}_{l}=\frac{1}{n} \sum_{i=1}^{n} x_{i l} .
$$

We know that attributes with small variations can provide more reliable information than those with large variations. Therefore, the attribute weight should be inversely related to its variation. It means that an attribute that has a large variation receives less weight than the attribute that has a smaller variation. Since attribute weights are considered to be non-negative with summation to one, they could be defined as the inverse of absolute $\mathrm{CV}$ values. However, we consider those applications to color image segmentation in which all data points are non-negative. Thus, the $l$ th attribute weight $w_{l}$ is proportional to $1 / c v_{l}$ and defined as

$$
w_{l}=\frac{1 / c v_{l}}{\sum_{t=1}^{p} 1 / c v_{t}}, j=1, \cdots, p .
$$

After the attribute weight is determined by the variation approach, the next step is to find the value of $\beta$ in the weighted mountain function Eq. (7). To find the suitable value of $\beta$, we use the synthetic data set shown in Table 1 with three normally distributed clusters in the two-dimentional subspace (see Fig. 3) and one noise variable (see Fig. 4). The 3D plots of the weighted mountain fountion Eq. (7) with $\beta=-5 \sim 5$ for each case 
are shown in Figs. 3.1 3.11 and Figs. 4.1 4.11, respectively. From these figures, we find that, in Figs. 3.5 and 4.5 with $\beta=-1$, the weighed mountain function gives a good density shape estimate whether the data set contains noise variables or not. Therefore, we take $\beta=-1$ in applications to color image segmentation

\section{Applications to color image segmentation}

For most image processing and computer vision algorithms, image segmentation is an important step. Thus, in this section, we compare the proposed method with Yang and $\mathrm{Wu}[6]$ with randomly generated initial cluster centers on color image segmentation. We use three color images shown in Figs. 5 7: butterfly with the size $127 \times 96$, clown with the size $128 \times 128$ from Kim et al. [4] and snoopy with the size $128 \times 96$. We set the parameters in W-k-means algorithm (see [2] as follows: (i) the termination criteria $\epsilon=0.0001$; (ii) the number of clusters $k=4$ in butterfly image, $k=8$ in clown image and $k=3$ in snoopy image. For simplicity, we choose the raw color data in the RGB color space. Thus, we run the W-k-means algorithm to the RGB space of these images with 10 sets of randomly generated initial cluster centers, the proposed feature-weighted mountain method and Yang and Wu's [6] method. The segmentation results of these images are shown in Figs. 5.1 7.12. Figures 5.1, 6.1 and 7.1 are segmentation results with the proposed approach. Figures 5.2, 6.2 and 7.2 are segmentation results with Yang and Wu's [6] method. Figures $5.3 \sim 5.12,6.3 \sim 6.12$ and $7.3 \sim 7.12$ are segmentation results using the W-k-means algorithm with the 10 sets of randomly generated initial cluster centers. To evaluate the results of color image segmentation, it is necessary for us to make a quantitative comparison of segmented images by different initial cluster centers in the proposed algorithm.

The following evaluation function $F(I)$ given by Liu and Yang [3] is used for our comparisons

$$
F(I)=\sqrt{R} \times \sum_{i=1}^{R} \frac{\left(e_{i} / 256\right)^{2}}{\sqrt{A_{i}}},
$$

where $I$ is the segmented image, $R$, the number of regions in the segmented image, $A_{i}$, the area, or the number of pixels of the $i$ th region, and $e_{i}$, the color error of region $i$. $e_{i}$ is defined as the sum of the Euclidean distance of the color vectors between the original image and the segmented image of each pixel in the region. In this paper, $R$ is equal to $k$. Note that the smaller the value of $F(I)$ is, the better the segmentation result should be. Figures $5.1 \sim 5.12,6.1 \sim 6.12$ and $7.1 \sim 7.12$ also show the values of $F(I)$ corresponding to segmented images. According to these values of $F(I)$ with segmented images, we find that the proposed approach (Fig. 5.1, Fig. 6.1 and Fig. 7.1) has better segmentation results than Yang and $\mathrm{Wu}$ [6] and the W-k-means algorith with randomly generated initial cluster centers.

\section{Conclusions}

We proposed a feature-weighted mountain clustering method so that it can work well for noisy feature variables. The proposed method can be also used for obtaining initial estimate of cluster centers for other clustering algorithms. Results from color image segmentation with evaluation function illustrate the proposed method actually produces better segmentation than previous methods.

\section{Acknowledgements}

The authors are grateful to Mr. Ming-Chieh Lin for his computing assistance. This work was supported in part by the National Science Council of the Republic of China under Grant NSC 98-2118-M-134-001.

\section{References}

[1] S.L. Chiu, Fuzzy model identification based on cluster estimation. J Intel Fuzzy Syst 2, 267-278, 1994.

[2] J.Z. Huang, M.K. Ng, H. Rong, and Z. Li, Automated variable weighting in $k$-means type clustering. IEEE Trans. Pattern Anal. Machine Intelligence 27, 657-668, 2005.

[3] J. Liu and Y.H. Yang, Multiresolution color image segmentation technique, IEEE Trans. on Pattern Analysis and Machine Intelligence 16, 689700, 1994.

[4] D.W. Kim, K.H. Lee, and D. Lee, A novel initialization for the fuzzy c-means algorithm for color clustering. Pattern Recognition Letters 25, 227237, 2004.

[5] B.W. Silverman, Density Estimation for Statistics and Data Analysis, Chapman \& Hall, New York, 1986.

[6] M.S. Yang and W.L. Wu, A modified mountain clustering algorithm, Pattern Anal. Applic. 8, 125-138, 2005.

[7] R.R. Yager and D.P. Filev, Approximate clustering via the mountain method. IEEE Trans. Syst. Man Cybern 24, 1279-1284, 1994 


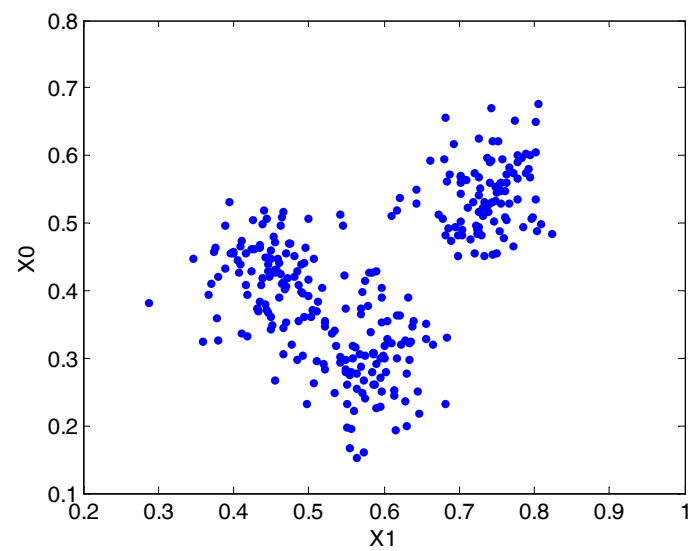

Fig. 1.1. The data set with three normally distributed clusters in the subspace of $(\mathrm{x} 0, \mathrm{x} 1)$.

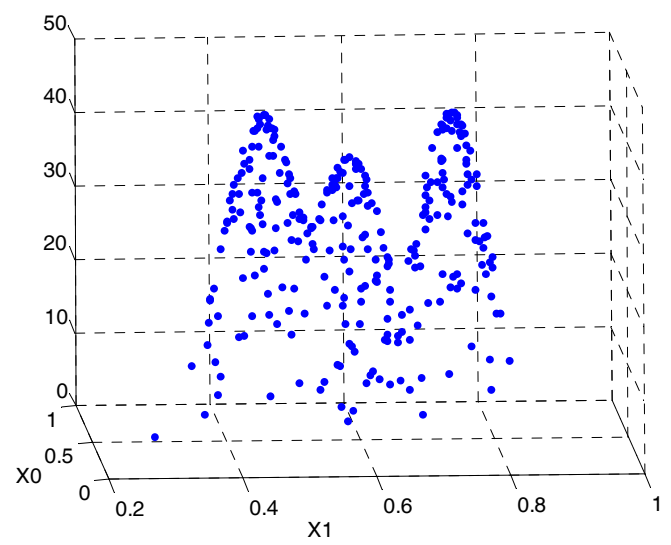

Fig. 1.2. The mountain function shows a good density shape estimate.

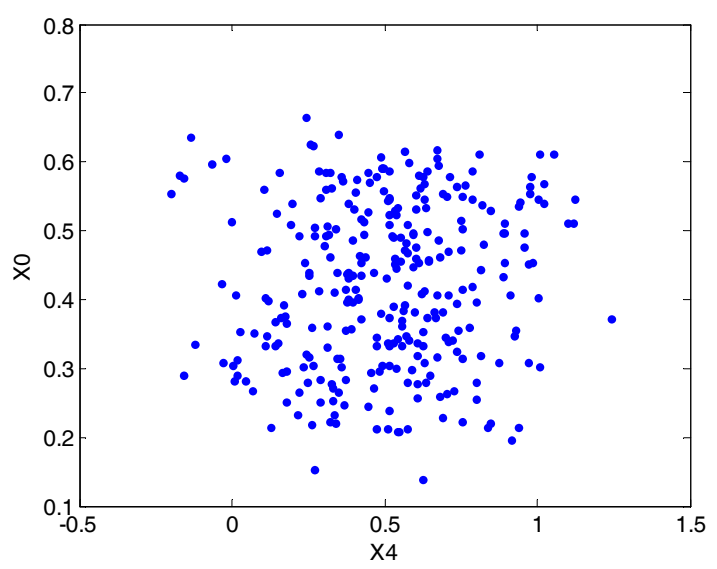

Fig. 2.1. The subspace of $(\mathrm{x} 0, \mathrm{x} 4)$ shows three clusters but it contains a noise variable.

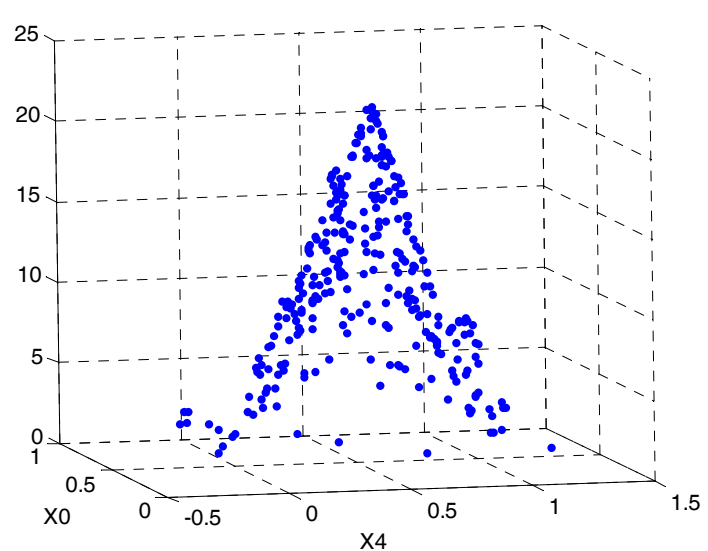

Fig. 2.2. A poor density shape estimate occurs when there exists some noise variable. 


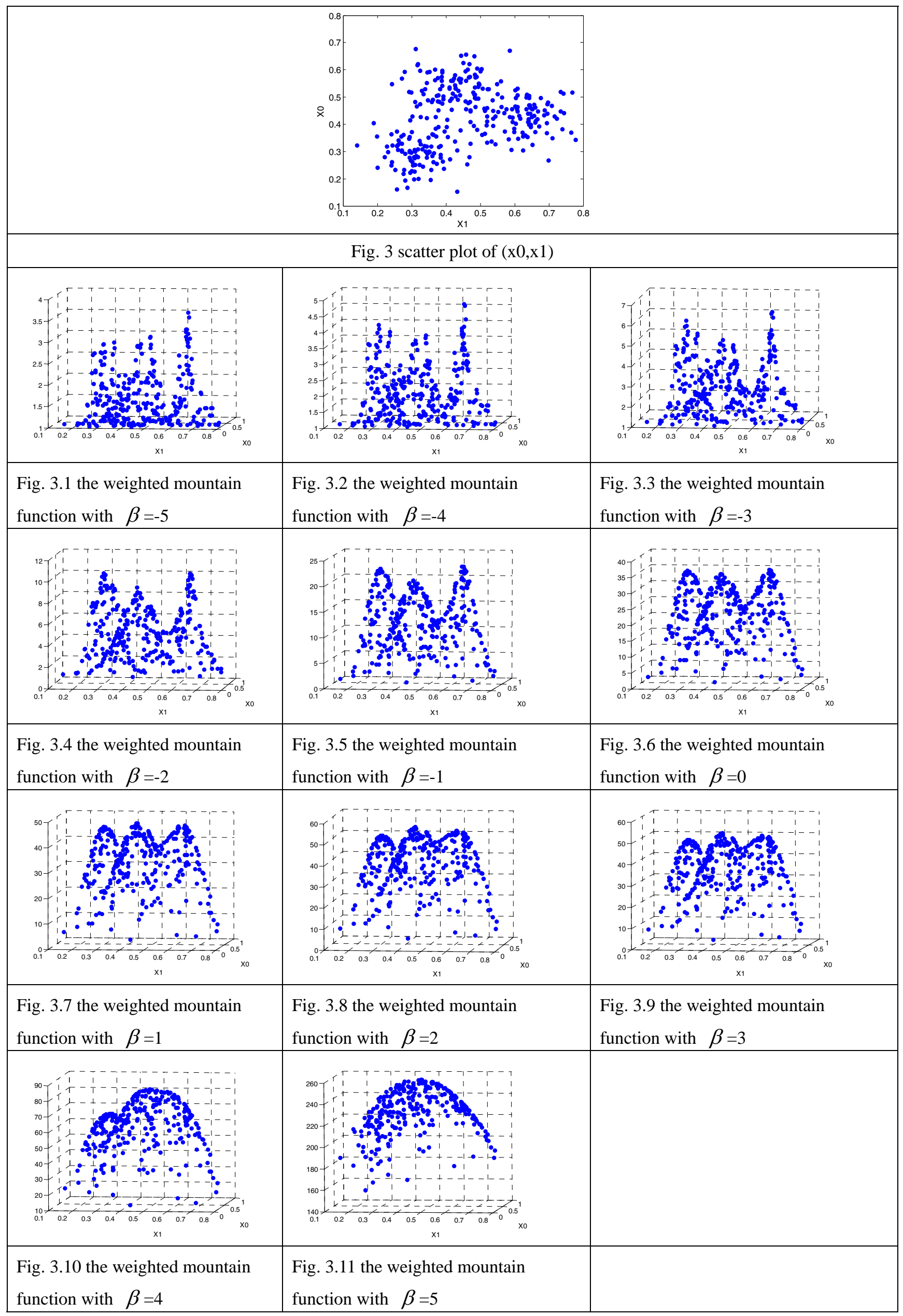




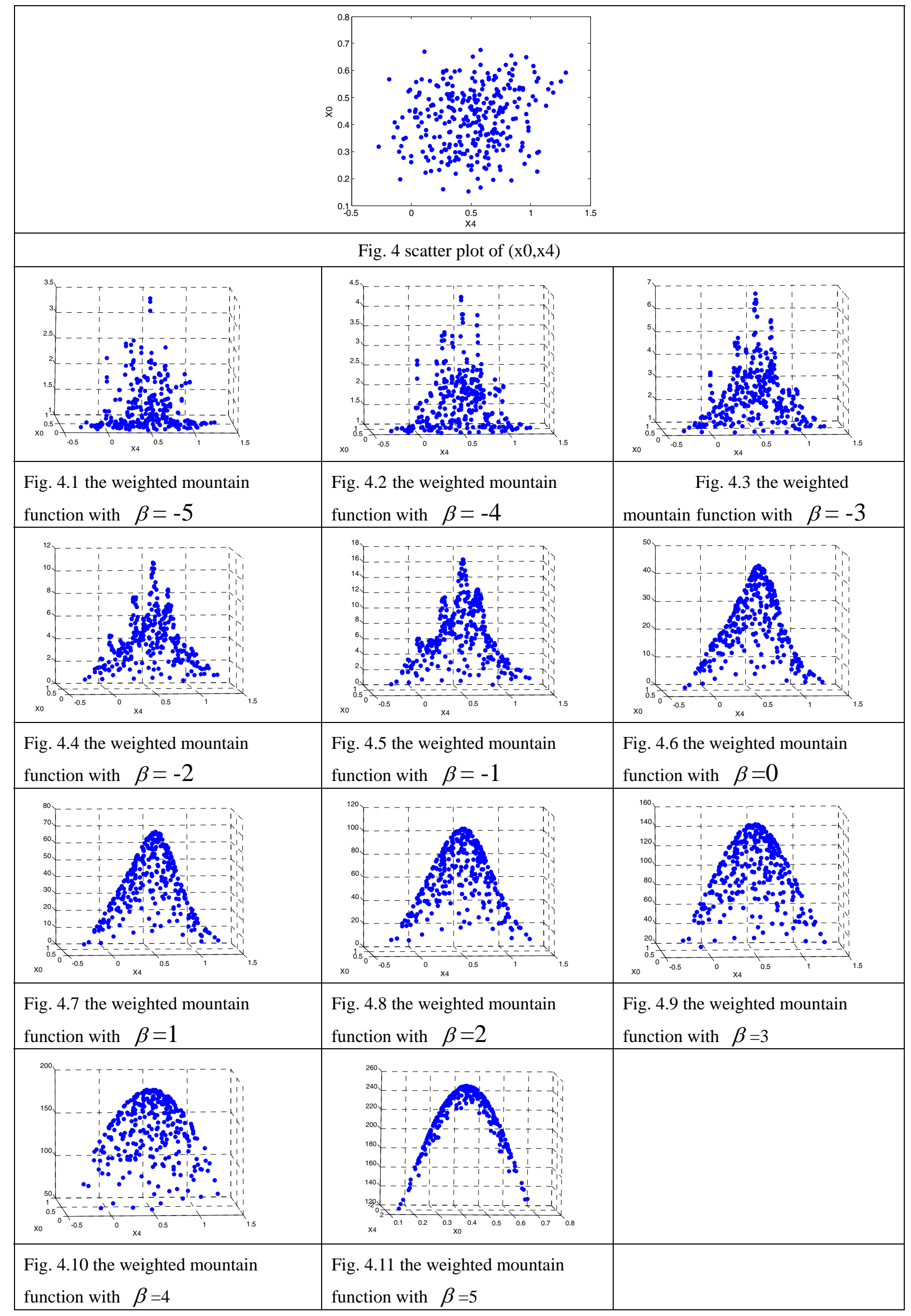




\begin{tabular}{|c|c|c|}
\hline \multicolumn{3}{|c|}{ Fig. 5 the original butterfly image } \\
\hline $\begin{array}{l}\text { Fig. } 5.1 \text { the segmentation result } \\
\text { of the proposed method, } \\
F(I)=\mathbf{6 . 7 9 9 9}\end{array}$ & $\begin{array}{l}\text { Fig. } 5.2 \text { the segmentation result } \\
\text { of Yang \& Wu's method, } \\
F(I)=6.8072)\end{array}$ & $\begin{array}{l}\text { Fig. } 5.3 \text { the segmentation result } \\
\text { of } \mathrm{W} \text {-k-means with randomlu } \\
\text { initial cluster centers } \\
\mathrm{F}(\mathrm{I})=20.9213 \text { ) }\end{array}$ \\
\hline $\begin{array}{l}\text { Fig. } 5.4 \text { the segmentation result } \\
\text { of } W \text {-k-means with randomly } \\
\text { initial cluster centers, } \\
\mathrm{F}(\mathrm{I})=\mathbf{6 . 7 9 9 9} \text { ) }\end{array}$ & $\begin{array}{l}\text { Fig. } 5.5 \text { the segmentation result } \\
\text { of } W \text {-k-means with randomly } \\
\text { initial cluster centers, } \\
\mathrm{F}(\mathrm{I})=20.9213 \text { ) }\end{array}$ & $\begin{array}{l}\text { Fig. } 5.6 \text { the segmentation result } \\
\text { of W-k-means with randomly } \\
\text { initial cluster centers, } \\
F(I)=6.8044)\end{array}$ \\
\hline $\begin{array}{l}\text { Fig. } 5.7 \text { the segmentation result } \\
\text { of } \mathrm{W} \text {-k-means with randomly } \\
\text { initial cluster centers, } \\
\mathrm{F}(\mathrm{I})=6.8072 \text { ) }\end{array}$ & $\begin{array}{l}\text { Fig. } 5.8 \text { the segmentation result } \\
\text { of } W \text {-k-means with randomly } \\
\text { initial cluster centers, } \\
\mathrm{F}(\mathrm{I})=20.9213 \text { ) }\end{array}$ & $\begin{array}{l}\text { Fig. } 5.9 \text { the segmentation result } \\
\text { of } W \text {-k-means with randomly } \\
\text { initial cluster centers, } \\
F(\mathrm{I})=20.9161 \text { ) }\end{array}$ \\
\hline $\begin{array}{l}\text { Fig. } 5.10 \text { the segmentation result } \\
\text { of } W \text {-k-means with randomly } \\
\text { initial cluster centers, } \\
F(I)=20.9161 \text { ) }\end{array}$ & $\begin{array}{l}\text { Fig. } 5.11 \text { the segmentation result } \\
\text { of } W \text {-k-means with randomly } \\
\text { initial cluster centers, } \\
\mathrm{F}(\mathrm{I})=6.8072 \text { ) }\end{array}$ & $\begin{array}{l}\text { Fig. } 5.12 \text { the segmentation result } \\
\text { of } W \text {-k-means with randomly } \\
\text { initial cluster centers, } \\
\mathrm{F}(\mathrm{I})=20.9213 \text { ) }\end{array}$ \\
\hline
\end{tabular}

Bold represents the smallest value 


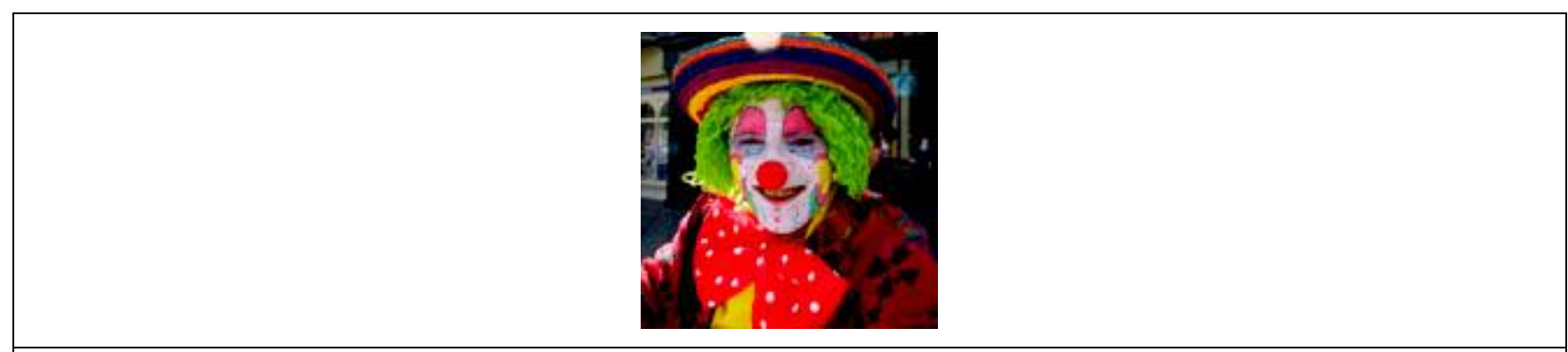

Fig. 6 the original clown image

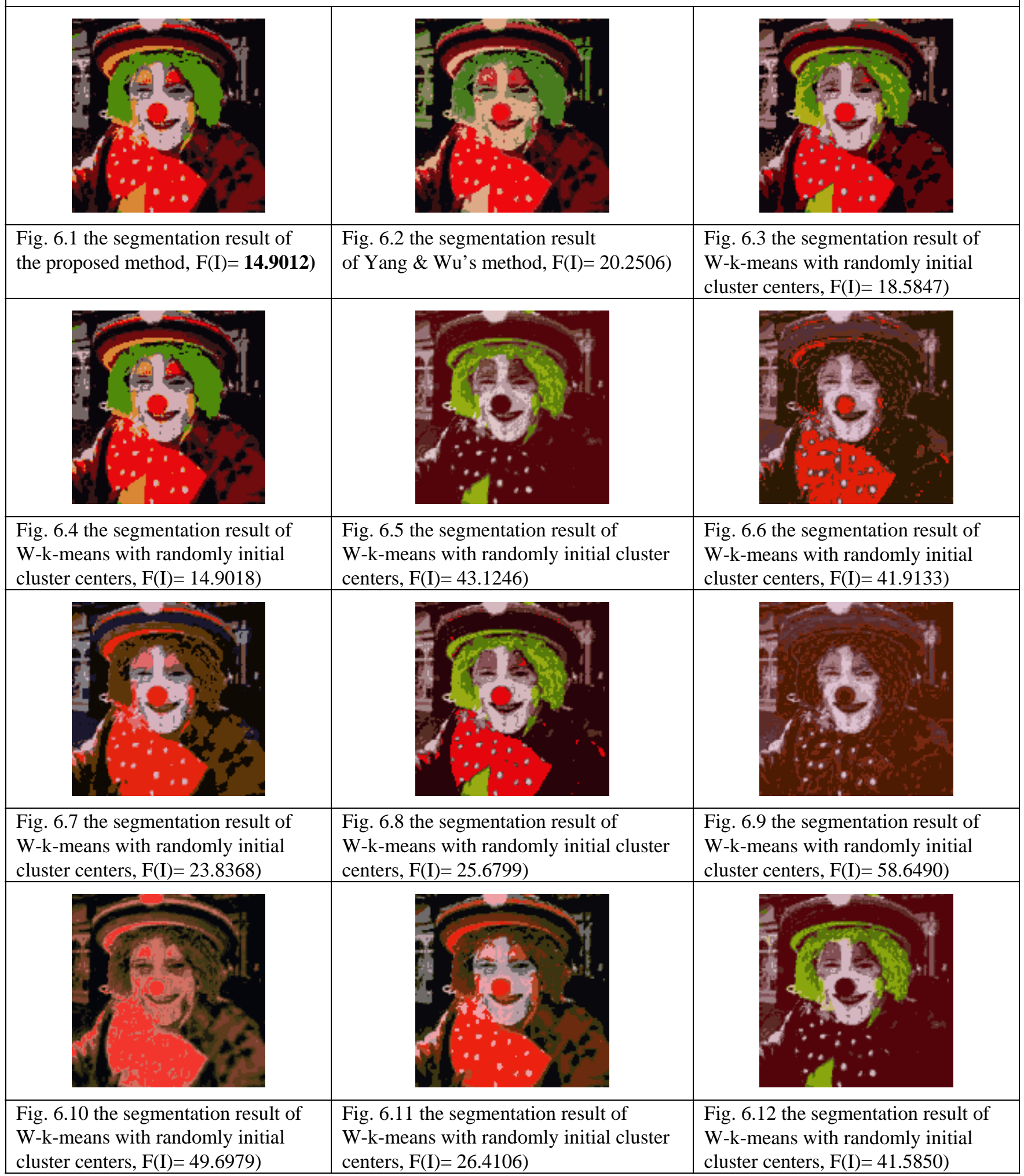

\section{Bold represents the smallest value}




\begin{tabular}{|c|c|c|}
\hline \multicolumn{3}{|c|}{ Fig. 7 the original snoopy image } \\
\hline $\begin{array}{l}\text { Fig. } 7.1 \text { the segmentation result } \\
\text { of the proposed method, } \\
F(I)=2.7514)\end{array}$ & $\begin{array}{l}\text { Fig. } 7.2 \text { the segmentation result } \\
\text { of Yang \& Wu's method, } \\
F(I)=2.7598)\end{array}$ & $\begin{array}{l}\text { Fig. } 7.3 \text { the segmentation result } \\
\text { of W-k-means with randomly } \\
\text { initial cluster centers, } \\
\mathrm{F}(\mathrm{I})=3.6509 \text { ) }\end{array}$ \\
\hline $\begin{array}{l}\text { Fig. } 7.4 \text { the segmentation result } \\
\text { of } W \text {-k-means with randomly } \\
\text { initial cluster centers, } \\
\mathrm{F}(\mathrm{I})=2.7598 \text { ) }\end{array}$ & $\begin{array}{l}\text { Fig. } 7.5 \text { the segmentation result } \\
\text { of } W \text {-k-means with randomly } \\
\text { initial cluster centers, } \\
\mathrm{F}(\mathrm{I})=3.6509)\end{array}$ & $\begin{array}{l}\text { Fig. } 7.6 \text { the segmentation result } \\
\text { of } W \text {-k-means with randomly } \\
\text { initial cluster centers, } \\
\mathrm{F}(\mathrm{I})=2.7598)\end{array}$ \\
\hline $\begin{array}{l}\text { Fig. } 7.7 \text { the segmentation result } \\
\text { of } W \text {-k-means with randomly } \\
\text { initial cluster centers, } \\
F(I)=2.7598 \text { ) }\end{array}$ & $\begin{array}{l}\text { Fig. } 7.8 \text { the segmentation result } \\
\text { of } W \text {-k-means with randomly } \\
\text { initial cluster centers, } \\
\mathrm{F}(\mathrm{I})=2.7598 \text { ) }\end{array}$ & $\begin{array}{l}\text { Fig. } 7.9 \text { the segmentation result } \\
\text { of W-k-means with randomly } \\
\text { initial cluster centers, } \\
\mathrm{F}(\mathrm{I})=3.6676)\end{array}$ \\
\hline $\begin{array}{l}\text { Fig. } 7.10 \text { the segmentation result } \\
\text { of } \mathrm{W} \text {-k-means with randomly } \\
\text { initial cluster centers, } \\
\mathrm{F}(\mathrm{I})=3.6676)\end{array}$ & $\begin{array}{l}\text { Fig. } 7.11 \text { the segmentation result } \\
\text { of W-k-means with randomly } \\
\text { initial cluster centers, } \\
\mathrm{F}(\mathrm{I})=2.7598)\end{array}$ & $\begin{array}{l}\text { Fig. } 7.12 \text { the segmentation result } \\
\text { of } \mathrm{W} \text {-k-means with randomly } \\
\text { initial cluster centers, } \\
\mathrm{F}(\mathrm{I})=3.6509 \text { ) }\end{array}$ \\
\hline
\end{tabular}

Bold represents the smallest value 